\title{
Actitudes y creencias hacia la discapacidad en clases de educación física. Una cuestión educativa
}

\author{
Juan GONZÁLEZ1 y Rosario CORTÉS
}

Universidad de Murcia, España

(Recibido 11 Abril, 2016; Aceptado 15 Junio, 2016)

RESUMEN: Hablar de discapacidad en la escuela, se trata de contemplar no sólo al estudiante que presenta deficiencias en su estructura física, sensorial o psíquica, sino que además vive en un contexto social que le obliga a interactuar con su medio para desempeñarse adaptado en las clases de educación física. La muestra ( $\mathrm{N}=71)$, recogida aleatoriamente entre estudiantes de educación secundaria, compañeros de alumnos con discapacidad, con edades entre 12 y 18 años ( $M=14.57$; DT=1.51). Con una metodología mixta, utilizando cuestionarios sobre cambio de actitudes y creencias hacia la discapacidad, y entrevistas a profesionales del entorno escolar $(\mathrm{N}=6)$, para contrastar la información obtenida en la parte cuantitativa. Aparecen altos valores de creencias hacia la discapacidad a favor de los chicos, mientras que las chicas muestran mayores actitudes hacia sus compañero/as discapacitado/as. De la misma forma, conforme aumentan las creencias hacia la integración, disminuyen el reconocimiento y la implicación, por lo que se reduce la intencionalidad.

Palabras clave: Inclusivo, integración, discapacidad, educación física, escuela.

Attitudes and beliefs towards disability in physical education classes. An educational question

ABSTRACT: To talk about disability at school, is contemplating students not only deficient in their physical, sensory or mental structure, but also their experiences in a social context that forces to interact adapted with their environment in classes of physical education. The sample $(\mathrm{N}=71)$, collected randomly among high school students, fellow students with disabilities, aged 12 and 18 years $(\mathrm{M}=14.57 ; \mathrm{SD}=1.51)$. With a mixed methodology, using questionnaires on changing attitudes and beliefs towards disability, and interviews with professionals $(\mathrm{N}=6)$ school environment, to contrast the information obtained in the quantitative part. Appear high values of beliefs about disability in favor of boys, while girls show higher attitudes toward their mates disabled/as. Appear High values of beliefs about disability in favor of boys, while girls show higher attitudes toward their mates disabled/as. Likewise, with increase the beliefs integration, students have less recognition and involvement, so the intent is reduced.

Keywords: Inclusive, integration, disability, physical education, school.

${ }^{1}$ Correspondencia: Dr. Juan González Hernández. Dpto. Personalidad, Evaluación y Tratamiento Psicológico. Facultad de Psicología. Universidad de Murcia. Campus de Espinardo, s/n. Espinardo. 30100 (Murcia). E-mail: jgonzalez@um.es. 
Referirse al término discapacidad, es referirse tanto por el exceso como por la insuficiencia de la realización de una actividad rutinaria, pudiendo tener un carácter temporal o permanente. Se trata del reflejo de una compleja relación entre la condición de salud de una persona, sus factores personales y factores externos que configuran las circunstancias en las que vive esa persona.

Ante tal dimensión, la discapacidad surge como consecuencia de la diferente respuesta del individuo a nivel psicológico ante deficiencias físicas, sensoriales o de otro tipo. Algunos autores señalan, que se entiende como toda restricción o ausencia (debido a una deficiencia) de la incapacidad de realizar una actividad dentro de lo que se considera "normal" para el ser humano (Cologon, 2013), aunque otros también relacionan el término a una delimitación del contexto en el que la persona se desenvuelve (Muñoz, Novo-Corti y Rebollo, 2013).

Educar en la diversidad significa ejercer los principios de igualdad y equidad a los que todo ser humano tiene derecho (An y Meaney, 2015; Madrid, Sánchez y García 2011), lo que conlleva desarrollar la implicación en el diseño y puesta en marcha de estrategias de enseñanza-aprendizaje, que personalicen la enseñanza en un marco y dinámica de trabajo para todos, así como de adaptaciones ambientales, con el propósito de que los alumnos se sientan incluidos en la vida del aula, del centro y de la comunidad a la que pertenecen(Calvo, PérezTejero y Löpez, 2015; Coates y Vickerman, 2013; Muntaner, 2000; Park, Koh, y Block, 2014) .

La literatura científica define la inclusión como una actitud, un valor y sistema de creencias, no sólo una acción o conjunto de acciones que abarcan a todos lo estudiantes (Tripp, Rizzo y Webert, 2007), sino a un sentimiento de pertenencia, bienestar emocional y relacional (Bebetsos,Derri, Filippou, Zetou, y Vernadakis, 2014; Qi y Ha, 2012), en torno a la construcción de actitudes y representaciones o creencias positivas en torno al aprendizaje y a las posibilidades de las personas con discapacidad (Gómez e Infante, 2004).

Por otra parte, la vida escolar en la que todos los alumnos deben sentirse incluidos, transcurre a través de las actividades de enseñanza y aprendizaje con sus iguales y no al margen de ellas (ya que la contribución de la educación escolar a la inclusión social de cualquiera, es poder alcanzar el mayor nivel de logro y de cualificación escolar posible). Dicho de otro modo, trabajar para la inclusión educativa es pensar en términos de las condiciones y procesos que favorecen un aprendizaje con significado y sentido para todos y todas (Echeita y Ainscow, 2004).

La actividad física como facilitadora de actitudes hacia la discapacidad

Centrados en el ámbito escolar, y especialmente en el de la actividad física, los planteamientos pedagógicos y docentes deben valorar tanto la interdependencia como la independencia (An y Meaney, 2015; Caus y Santos, 2011, González y Baños, 2012), entendiendo la diversidad del alumnado como una oportunidad para favorecerse la integración e inclusión, generando y potenciando actitudes activas a través de la gran variedad de oportunidades que ofrecen las clases de actividad física (juegos, ejercicios y actividades, etc.) (Futornyi, 2013).

La EFD (Educación Física Deportiva) tiene como objetivo la educación de las conductas motrices; pone en juego al cuerpo, las capacidades específicas desarrolladas a través de 
capacidades físicas, y por otra parte, al desarrollo de las capacidades cognitivas, afectivas y relaciones de la persona (Pierre, 2007).

Desde la parte docente, las ventajas de una práctica inclusiva en educación física (Braga, Tracy y Taliaferro, 2015; DePauw y Doll-Tepper, 2000), se convierten en los beneficios propios de la práctica de actividad física, añadiendo los producidos psicosocialmente por organizar las sesiones desde la diversidad del alumnado.

Algunos trabajos analizan el impacto de la inclusión en las sesiones de educación física (Bossaert, Colpin, Pijl, y Petry, 2013; Ozer et al., 2013; Place y Hodge, 2001), constatando que incluir estudiantes con o sin discapacidad en las clases de educación física ayuda a incrementaría la interacción social, sólo si el proceso inclusivo se produce con adaptaciones curriculares apropiadas, modificando las instrucciones y reglamentos, incorporando recursos humanos (tutores, especialistas, entre otros) y tomando decisiones sobre el terreno según sea la sesión (ver cuadro1). En este sentido, los alumnos con discapacidad prefieren la inclusión en las clases de educación física, dado que les proporciona la oportunidad de beneficiarse de la realización de mayor número y diversidad de actividades y de compartir en mayor medida con sus compañeros sin discapacidad (An y Meaney, 2015; Goodwin y Watkinson, 2000), en lugar de otros planteamientos que han supuesto barreras o limitaciones para el desarrollo e inclusión (Bebetsos et al., 2014).

- La oportunidad de desarrollar habilidades sociales necesarias para la interacción con los otros.

- La oportunidad de desarrollar amistades entre compañeros con y sin discapacidad.

- La oportunidad de interactuar con modelos adecuados a su edad.

- Se disminuye el aislamiento y se incrementan las expectativas de la persona.

- Cambia la actitud ante los iguales, incrementándose la aceptación de la diferencia.

- Mayor compresión de los derechos de la discapacidad y la equidad.

Cuadro 1. Estrategias docentes para el planteamiento de la EFD con mayor inclusividad

En el proceso de inclusión del discapacitado al ámbito educativo, y siempre teniendo en cuenta las necesidades de la discapacidad concreta (sensorial, física, psíquica,...), podría hablarse de un proceso a varios niveles: a) un primer nivel de accesibilidad, en el que deben existir (o generarse) las infraestructuras necesarias para la adaptación del discapacitado; b) un segundo nivel de adaptación curricular; en el que la metodología pedagógica debe plantearse de forma que el discapacitado adquiera los contenidos básicos de cada etapa educativa, y c) un tercer nivel de facilitación social; en el que debe establecerse un sistema de constante sociabilidad entre los miembros de la comunidad educativa, que incluye los procesos de integración, generación de actitudes de comprensión y de prosocialidad.

Tomando como referencia dos de los aspectos claves del presente estudio, trabajos anteriores (Sullivan y Masters, 2014; Molina y Valenciano, 2010) señalan que las creencias y las actitudes son dos constructos interrelacionados, y así lo demuestran en un estudio de caso realizado hacia un profesor de educación física con discapacidad, donde ponen de manifiesto tal teoría. Entre las múltiples definiciones existentes sobre las actitudes, la más clarificadora concluye que "la actitud es una predisposición aprendida para responder constantemente de un modo favorable ante objetos, personas o grupos de personas y situaciones" (Alves y Duarte, 2014; Ko y Boswell, 2013; Obrusnikova y Dillon, 2012). 
Teniendo en cuenta esta definición, la evidencia científica indica que las actitudes que se tengan hacia las personas influirán en la interacción con ellas (figura 1), de forma que en función de la concepción que tengamos sobre las personas con discapacidad así será nuestra predisposición a comportarnos en nuestras relaciones con ellas, y con ello a influir en su integración en la sociedad (Aguado, Alcedo, y Arias, 2008; Bossaert, Colpin, Pijl y Petry, 2010; Florez, Aguado y Alcedo, 2009; González y Baños, 2012).

Se considera que las actitudes son una de las variables más importantes a la hora de facilitar o dificultar el proceso de integración e inclusión de los alumnos con discapacidad en el ámbito educativo (Coates y Vickerman, 2013; Konur 2006; Novo-Corti, Muñoz-Cantero y Calvo-Porral, 2011; Sullivan y Masters, 2014). Por tanto, es conveniente examinar las actitudes hacia los estudiantes con discapacidad en sus diferentes etapas educativas para poder realizar una intervención para modificarlas o mejorarlas en la medida que sea posible (Araya-Cortés, González-Arias, y Cerpa-Reyes, 2014; Gómez e Infante, 2004; Hammond et al, 2014).

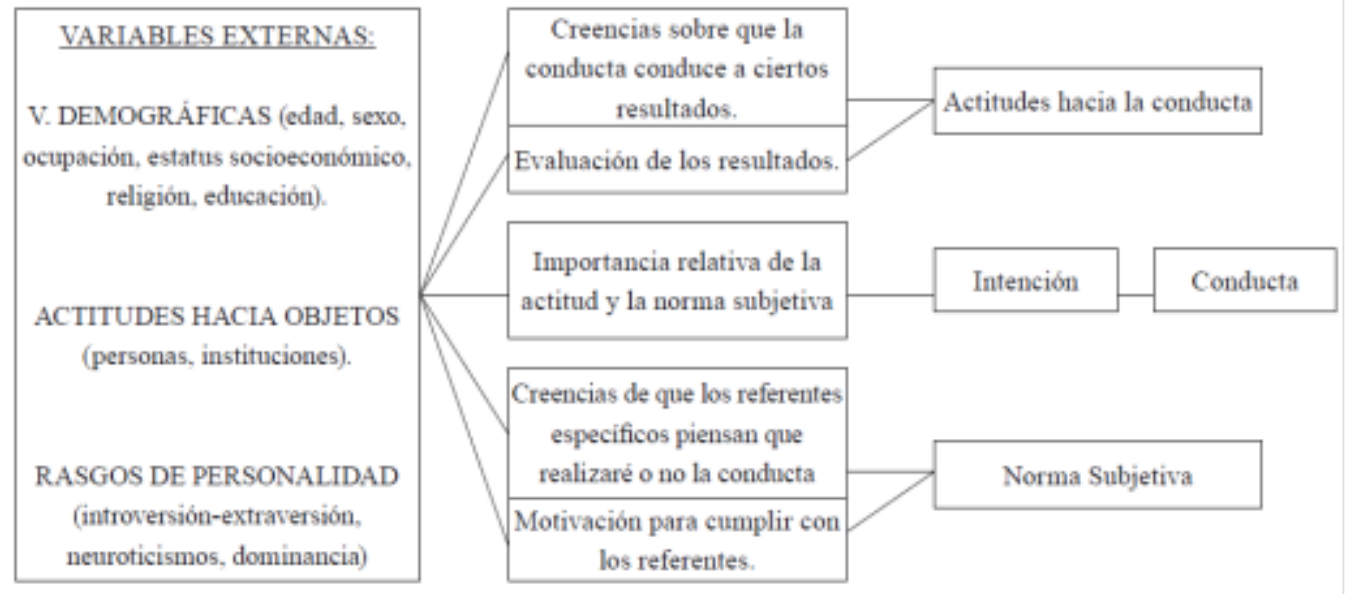

Figura 1. Modelo para la formación de actitudes de Fisbein y Ajzen (1984) (en Novo-Corti et al., 2011)

El presente estudio pretende como objetivo principal describir el grado de concienciación de los iguales, acerca de la participación de la discapacidad en las dinámicas de las clases de actividad física. Tras la revisión bibliográfica realizada, se plantea como hipótesis de estudio que las actitudes de apoyo y valoración facilitarán la intencionalidad para la atención a la discapacidad en los iguales, y que los responsables del entorno educativo indicarán la idoneidad de propuestas de integración para instaurar actitudes y creencias hacia la discapacidad en los alumnos, y que conforme aumente la capacidad actitudinal, aumentarán las creencias hacia la discapacidad de los iguales.

\section{Método}

Para el presente trabajo se ha utilizado una metodología mixta (figura 2), tanto cuantitativa (recogida muestral entre los iguales-compañeros de niños con discapacidad) como cualitativa (entrevistas a personal docente de educación física y personal de apoyo). 


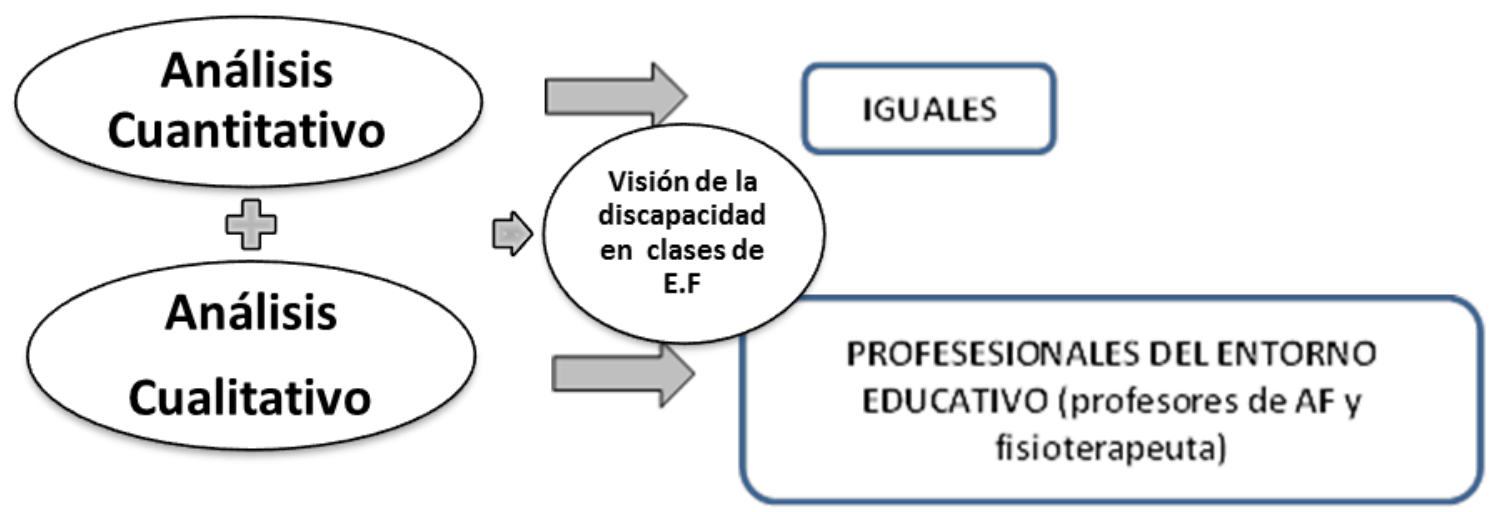

Figura 2. Descripción de la metodología mixta llevada a cabo

\section{Participantes}

En el total de este estudio han participado 71 estudiantes (33 chicas y 38 chicos); con edades comprendidas entre 12 y 18 años ( $\mathrm{M=14.07;} \mathrm{DT=1.01),} \mathrm{cursando} \mathrm{ESO} \mathrm{o} \mathrm{Bachillerato} \mathrm{en}$ dos institutos de la Región de Murcia; todos ellos con algún compañero discapacitado motriz o auditivo (Tabla 1). Así como 5 profesores de A.F que imparten clase a los alumnos mencionados, tanto a los discapacitados como a sus iguales, y una fisioterapeuta encargada de la supervisión y/o colaboración con una de las alumnas discapacitadas.

Tabla 1. Estadísticos descriptivos de la muestra según sexo, género y convivencia con alumnos con discapacidad

\begin{tabular}{|c|c|c|c|c|c|c|c|}
\hline & \multicolumn{2}{|c|}{ Género } & \multirow[b]{2}{*}{ Total } & \multicolumn{4}{|c|}{ Convivencia con discapacitado } \\
\hline & chicas & chicos & & 1año & 2años & 3años & 4años \\
\hline $2^{\circ} \mathrm{ESO}$ & 10 & 12 & $22(31 \%)$ & 8 & 12 & 2 & \\
\hline $3^{\circ} \mathrm{ESO}$ & 13 & 17 & 30 (42.3\%) & 14 & 9 & & 7 \\
\hline $4^{\circ} \mathrm{ESO}$ & 10 & 9 & 19 (26.8\%) & 5 & 11 & 1 & 2 \\
\hline $\mathrm{N}=71$ & 33 & 38 & & 27 & 32 & 3 & 9 \\
\hline & (46.5\%) & (53.5\%) & & (38.03\%) & (45.07\%) & (4.22\%) & (12.68\%) \\
\hline
\end{tabular}

Debe reflejarse que un $38.03 \%$ de la muestra ha compartido tiempo de escuela con algún alumno discapacitado al menos durante 1 año, mientras que el resto de participantes indican que han compartido tiempo escolar con iguales discapacitados más de dos años (45.07\%), más de 3 (4.22\%) y más de 4 años (12.68\%). En cuanto al curso en el que los iguales han coincidido con los alumnos discapacitados. Se observa en la tabla 1 que un $31 \%$ de la muestra encuestada estaba cursando $1^{\circ}$ E.S.O, un $42.3 \% 3^{\circ}$ E.S.O y por último un $26.8 \%$ en $4^{\circ}$ de E.S.O. 


\section{Instrumentos}

-Cuestionario Sociodemográfico: Creado ad hoc, considerando variables como sexo, edad, curso, lugar de procedencia y tiempo de contacto con alguien con discapacidad.

-Escala de Actitudes hacia las Personas con Discapacidad, Forma G (EAPD; Verdugo, Arias y Jenaro, 1994): Se trata de una escala de evaluación de actitudes ante personas con cualquier tipo de discapacidad. Formada por 37 ítems, los cuales integran cinco sub-escalas: Valoración de capacidades y limitaciones (VCL); Reconocimiento/negación de los derechos (R-ND); Implicación personal (IP); Calificación genérica (CG) y Asunción de roles (AR) .La fiabilidad interna de la escala es de .79.

-Escala de Creencias hacia la atención a la discapacidad en actividad física (ECAF; González y Baños, 2012).Para la evaluación de los elementos actitudinales se elaboró un autoinforme ad hoc centrado en las creencias que mueven el cambio comportamental. Dicho autoinforme fue testado previamente por cinco expertos que facilitaron la orientación de los ítems a cada una de las subescalas resultantes, que han sido las siguientes: Creencias de control percibido ("Me sentiría incómodo trabajando con compañeros minusválidos o discapacitados"); Creencias sobre la actitud ("Sobre discapacidad preferiría que me asesorara un especialista a un compañero), Creencias generalizadas ("los alumnos con discapacidad se sienten más cómodos en las aulas de Educación Especial”),y Creencias intencionales ("Preferiría no tener alumnos con discapacidad en mi clase”).Analizada la consistencia de la escala, se obtiene un alpha de cronbach de .81 .

-Análisis cualitativo (Entrevista). Para el análisis e interpretación de los datos cualitativos se escogieron diferentes categorías atendiendo los temas principales planteados en la investigación: Actitudes y creencias respecto a la discapacidad hacia alumnos discapacitados por parte de sus iguales.

\section{Procedimiento}

Se ha accedido a la muestra tras la aprobación por parte del Equipo Directivo de cada centro a realizar la investigación y la posterior conformidad del profesorado en cuestión, por lo que no ha sido necesario firmar un consentimiento informado. A pesar de ser de carácter voluntario, el $100 \%$ del alumnado ha decidido participar en ella. Se han pasado los cuestionarios a todos los alumnos en clase de educación física. Las encuestas fueron administradas a los alumnos en cuatro sesiones, una por cada curso y/o clase que participó. A continuación se procedió a la elaboración de las entrevistas que posteriormente se han entregado a diferentes profesores de Educación Física y a una fisioterapeuta que cuentan como apoyo con algún alumno discapacitado. En ellas, con total disponibilidad, los profesores contestan a las preguntas planteadas con total libertad y anonimato, aportando su visión y opinión al respecto. Dichas entrevistas se les realizaron fuera de clase, pero dentro del Centro. 


\section{Análisis estadístico}

Se realizan los pertinentes análisis descriptivos (medidas de tendencia central) y exploratorio, estudio comparativo mediante pruebas t de contraste de hipótesis (ANOVA), y análisis correlacional entre las variables objeto de estudio. La metodología cuantitativa, fue analizada mediante el paquete estadístico SPSS 20.0. De la misma forma, los datos recogidos en las entrevistas cualitativas, se han determinado en el programa estadístico ATLAS.TII 6.2., que ha permitido el análisis de la información recogida durante la investigación y presentar los resultados en función de los objetivos propuestos.

\section{Resultados}

\section{Análisis cuantitativo}

En la Tabla 2 se señalan los estadísticos de contraste entre chicos y chicas compañeros de alumnos discapacitados; en la misma aparecen diferencias significativas a favor de las chicas en la valoración de capacidades y límites, implicación personal (tendencia a la significación) y genéricas; y a favor de los chicos en las creencias actitudes y de generalización.

Tabla 2. Estadísticos de grupo para el variable género

\begin{tabular}{|c|c|c|c|c|c|}
\hline & nero & Media & DT. & $\begin{array}{l}\text { Error típ. } \\
\text { de la media }\end{array}$ & Prueba t $\left(\mathrm{t}_{69,2)}\right.$ \\
\hline \multirow[t]{2}{*}{ VCL } & chicas & 3.11 & .646 & .112 & $2.035 *(.046)$ \\
\hline & chicos & 2.78 & .712 & .115 & \\
\hline \multirow[t]{2}{*}{ RND } & chicas & 2.59 & .919 & .160 & \\
\hline & chicos & 2.21 & .992 & .160 & \\
\hline \multirow[t]{2}{*}{ IP } & chicas & 2.51 & 1.052 & .183 & $1.924^{\dagger}(.058)$ \\
\hline & chicos & 2.04 & .988 & .160 & \\
\hline \multirow[t]{2}{*}{ CG } & chicas & 3.26 & 1.024 & .178 & $2.293 *(.025)$ \\
\hline & chicos & 2.71 & .987 & .160 & \\
\hline \multirow[t]{2}{*}{ AR } & chicas & 2.93 & 1.223 & .213 & \\
\hline & chicos & 2.71 & 1.261 & .204 & \\
\hline \multirow[t]{2}{*}{ CONTPER } & chicas & 3.35 & 1.032 & .179 & \\
\hline & chicos & 3.69 & .936 & 151 & \\
\hline \multirow[t]{2}{*}{ ACTITUD } & chicas & 3.06 & .427 & .074 & $-2.097 *(.040)$ \\
\hline & chicos & 3.29 & .498 & .081 & \\
\hline \multirow[t]{2}{*}{ GENERALIZ } & chicas & 2.90 & .463 & .081 & $-3.463 * *(.001)$ \\
\hline & chicos & 3.31 & .529 & .086 & \\
\hline INTENCIO & chicas & 3.03 & .771 & .134 & \\
\hline \multicolumn{6}{|c|}{$\mathrm{N}=71$ ( $\mathrm{n}$ chicos=38; $\mathrm{n}$ chicas= 33 ) } \\
\hline
\end{tabular}

*significativa si $\mathrm{p}<.05$; ** significativa si $\mathrm{p}<.01$ 
La distribución en el desarrollo de actitudes a lo largo de los años de convivencia con compañeros con discapacidad indica (Figura 3), indica que conforme aumentan los años, las actitudes experimentan un leve aumento con respecto al inicio de la convivencia, llegando al tercer año como máximo en todas ellas. A partir de este momento, coincidiendo con los 15-16 años, donde se produce un evidente descenso de todas y cada una de las actitudes que se miden (valoración, reconocimiento, implicación personal, genéricas y asunción de roles).

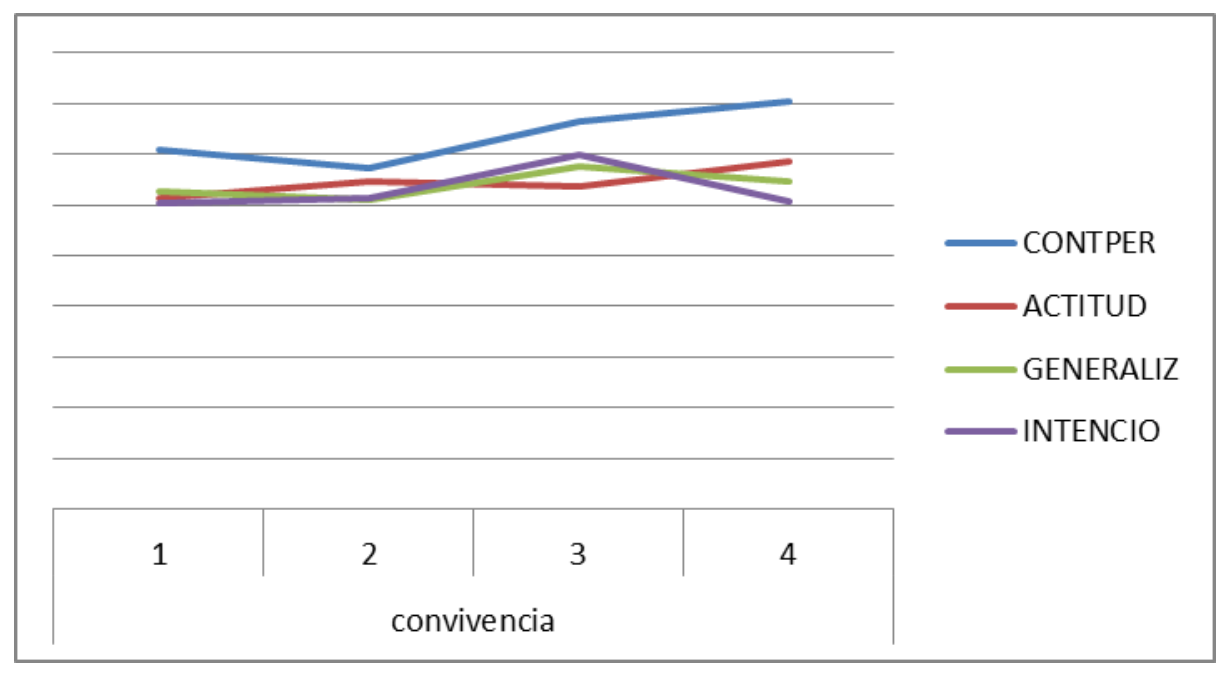

Figura 3. Actitudes de los iguales a lo largo de los años de convivencia con compañeros con discapacidad.

Con respecto a la distribución de las creencias (figura 4), se evidencia que, a lo largo de los años de convivencia con compañeros con discapacidad, conforme aumenta el tiempo, las creencias tienden a sufrir un leve aumento con respecto a las iniciales (primer año). De forma más específica, algunas creencias experimentan cambios más evidentes en el proceso. Las creencias de intencionalidad aumentan al tercer año pero sufren una caída al cuarto año y las creencias actitudinales experimentan un aumento más evidente hacia el $4^{\circ}$ año. Son las creencias acerca del control personal hacia la integración al compañero discapacitado las que se muestran más altas en todo momento.

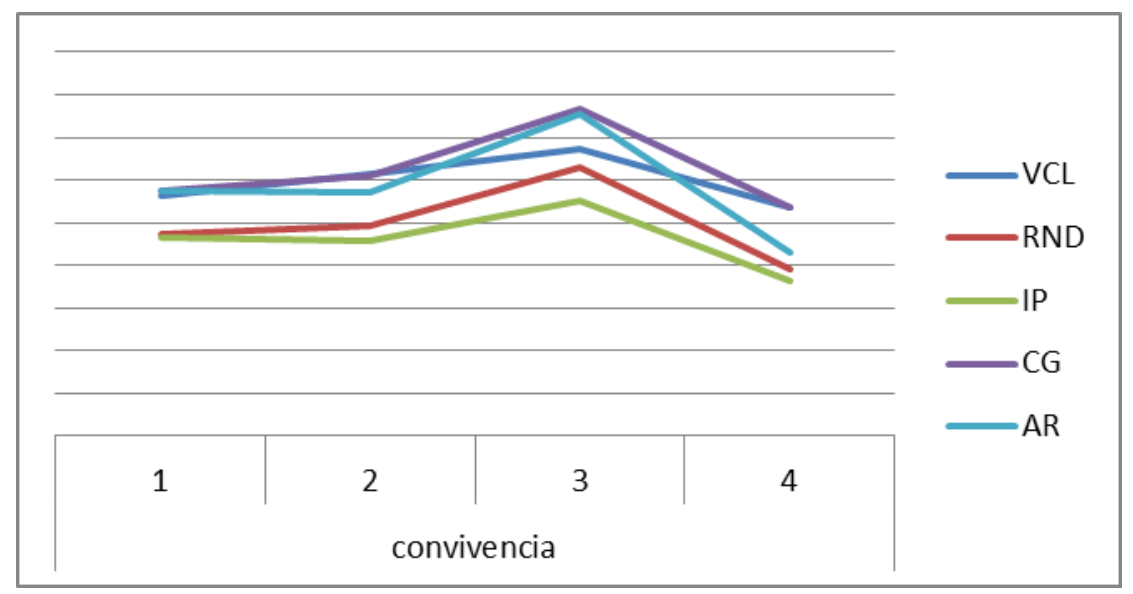

Figura 4. Creencias de iguales a lo largo de los años de convivencia con compañeros con discapacidad. (c) Psy, Soc, \& Educ, 2016, Vol. 8(2) 
La matriz de correlaciones entre las variables de estudio (Tabla 3), muestra que existe una linealidad inversa entre todas las creencias y actitudes estudiadas; más específicamente, aparecen índices de correlación negativos y medios entre la creencia de control personal y las actitudes, de manera que conforme aumenta la creencia de control personal hacia la integración de personas con discapacidad, disminuyen las actitudes de valoración, reconocimiento, implicación personal, genéricas y asunción de rol.

Tabla 3. Matriz de correlaciones entre actitudes y creencias hacia la discapacidad

\begin{tabular}{lccccc}
\hline & VCL & RND & IP & CG & AR \\
\hline CONTPER & $-.363^{* *}(.002)$ & $-.462^{* *}(.000)$ & $-.550^{* *}(.000)$ & $-.368^{* *}(.002)$ & $-.332^{* *}(.005)$ \\
ACTITUD & $-.249^{*}(.036)$ & $-.359^{* *}(.002)$ & $-.428^{* *}(.000)$ & & $-.308^{* *}(.009)$ \\
GENERALIZ & & $-.385^{* *}(.001)$ & $-.372^{* *}(.001)$ & $-.343^{* *}(.003)$ & $-.288^{*}(.015)$ \\
INTENCIO & $-.261^{*}(.028)$ & $-.298^{*}(.011)$ & $-.295^{*}(.012)$ & & \\
N= 71 & & & & & \\
\hline
\end{tabular}

*significativa si $p<.05 ;{ }^{* *}$ significativa si $p<.01$

De la misma forma, se evidencia que conforme aumenta la creencia de tener actitud hacia la integración de personas con discapacidad, disminuyen las actitudes de valoración, reconocimiento, implicación personal y la de asunción de rol. Además, las creencias de generalización correlacionan inversamente con las actitudes de reconocimiento, implicación personal, genéricas y la de asunción de rol. Y las creencias de intencionalidad, correlacionan inversamente con las actitudes de valoración, reconocimiento e implicación personal.

\section{Análisis cualitativo}

Tras las 6 entrevistas realizadas, las valoraciones que transmitieron tanto a los profesores de educación física como fisioterapeuta, se reflejan en la Tabla 4 las categorías establecidas acerca de actitudes y creencias, así como la discriminación y descripción de las subcategorías respondiendo a indicadores aportados por los distintos informantes y vinculados directamente con los temas principales seleccionados de antemano.

Las principales razones por las que reconocen que la variable VCL (valoración de las capacidades y limitaciones) tiene una presencia notable en las clases de A.F, es por la falta de comunicación, de materiales y /o recursos. A su vez, la mayoría de los entrevistados están de acuerdo que todo dependerá del tipo de discapacidad que presente el alumno, así como del tipo de actividad que realice. Por lo que no tendrán las mismas limitaciones en clases de educación física un alumno con discapacidad motriz, que otro con discapacidad auditiva (ya que son los dos tipos de discapacidades con las que se han trabajado).

En cuanto a la variable RND (reconocimiento/negación de los derechos), en todas y cada una de las entrevistas coinciden en que deben existir los mismos derechos tanto para 
alumnos discapacitados como para los que no lo son. Reconociéndose como un derecho imprescindible y que no debe ser vulnerado.

Tabla 4. Categorías y Subcategorías establecidas en el análisis cualitativo

\begin{tabular}{|c|c|c|}
\hline CATEGORIAS & SUBCATEGORÍAS & DEFINICIÓN \\
\hline \multirow[t]{6}{*}{ Actitudes } & $\begin{array}{l}\text { Valoración de las capacidades y } \\
\text { limitaciones } \\
\text { VCL(1) }\end{array}$ & $\begin{array}{l}\text { Su contenido se refiere esencialmente a la concepción que } \\
\text { el sujeto tiene de las personas con discapacidad respecto a } \\
\text { su capacidad de aprendizaje y de desempeño, y muestra las } \\
\text { inferencias sobre aptitudes (generales y específicas) } \\
\text { orientadas a la ejecución de tareas. }\end{array}$ \\
\hline & $\begin{array}{l}\text { Rendimiento/Negación de los } \\
\text { derechos } \\
\operatorname{RND}(2)\end{array}$ & $\begin{array}{l}\text { Sus contenidos se refieren al reconocimiento de derechos } \\
\text { fundamentales de la persona (por ejemplo, igualdad de } \\
\text { oportunidades, votar, acceder a créditos, etc.) y, en } \\
\text { particular, al derecho que tiene a la normalización y a la } \\
\text { integración social. }\end{array}$ \\
\hline & $\begin{array}{l}\text { Implicación personal } \\
\text { IP(3) }\end{array}$ & $\begin{array}{l}\text { Está formado por juicios referidos a comportamientos } \\
\text { concretos de interacción que la persona llevaría a efecto en } \\
\text { relación con personas con discapacidad, ya sea en } \\
\text { situaciones personales, laborales y/o sociales. }\end{array}$ \\
\hline & $\begin{array}{l}\text { Calificación genérica } \\
\text { CG(4) }\end{array}$ & $\begin{array}{l}\text { Se compone de atribuciones globales y calificaciones } \\
\text { genéricas que la persona efectúa acerca de rasgos } \\
\text { presuntamente definitorios de la personalidad o conducta } \\
\text { de las personas con discapacidad. }\end{array}$ \\
\hline & $\begin{array}{l}\text { Asunción de roles } \\
\text { AR(5) }\end{array}$ & $\begin{array}{l}\text { Agrupa ítems que consisten en presunciones que el sujeto } \\
\text { efectúa acerca de la concepción que de sí mismas tienen las } \\
\text { personas con discapacidad (por ejemplo, autoconfianza, } \\
\text { capacidad de normalización social, satisfacción consigo } \\
\text { mismas, autoestima elevada). }\end{array}$ \\
\hline & $\begin{array}{l}\text { Control percibido } \\
\mathrm{CP}(6)\end{array}$ & $\begin{array}{l}\text { Capacidad de control del individuo, tal y cómo es percibida } \\
\text { por él mismo.Va a facilitar la adaptación emocional ante } \\
\text { cualquier situación amenazante, ayudando a las personas a } \\
\text { poner en marcha estrategias activas de afrontamiento }\end{array}$ \\
\hline \multirow[t]{2}{*}{ Creencias } & $\begin{array}{l}\text { Intencionales } \\
\text { INTEN(7) }\end{array}$ & $\begin{array}{l}\text { La intención es el estado de la disposición mental que se } \\
\text { organiza a partir de las vivencias y que orienta o dirige la } \\
\text { respuesta de un sujeto ante determinados acontecimientos. } \\
\text { Forma en la que un individuo se adapta de forma activa a } \\
\text { su entorno y es la consecuencia de un proceso cognitivo, } \\
\text { afectivo y conductual. }\end{array}$ \\
\hline & $\begin{array}{l}\text { Generalizadas } \\
\text { GEN(8) }\end{array}$ & $\begin{array}{l}\text { Se podría definir como la percepción de la habilidad propia } \\
\text { para interactuar eficazmente con el entorno. }\end{array}$ \\
\hline
\end{tabular}

La IP (implicación personal), que según los entrevistados es facilitadora por parte de los centros, y en caso de ellos como profesores es muy positiva y favorecedora, ya que en todo momento quieren y/o intentan implicarse al máximo para favorecer esa integración de los alumnos discapacitados con el resto de compañeros, considerados sus iguales, en las clases de educación física. La CG (calificación genérica) respecto de si la educación física es favorecedora para los alumnos discapacitados, viene a indicar que es algo que logra motivar mucho en la gran mayoría de casos a los alumnos discapacitados y que es necesario que estos 
alumnos formen parte de los centros ordinarios, salvo excepciones de aquellas discapacidades más severas, para poder mantener esa motivación y lograr la consecución de esa integración.

Respecto a la AR (asunción de roles), el análisis cualitativo determina que es necesario un apoyo externo a los profesores de AF para poder conseguir que sus clases sean lo más adaptadas posibles a los alumnos discapacitados. De ahí la importancia de la figura de un fisioterapeuta, o un cuidador, que estén con los alumnos durante el desarrollo de las actividades en clase. La siguiente variable hace referencia al control percibido, CP, la cual determina que en la mayoría de casos de discapacidad, los propios alumnos discapacitados son conscientes de su discapacidad y de sus limitaciones, acentuándose con el paso de los años. En cuanto al resto de alumnos, considerados sus iguales, los entrevistados están de acuerdo en que cuanto más conviven con ellos, más conscientes son de las discapacidades que tienen.

Tabla 5. Análisis cualitativo de las entrevistas sobre creencias y actitudes hacia la discapacidad en educación física

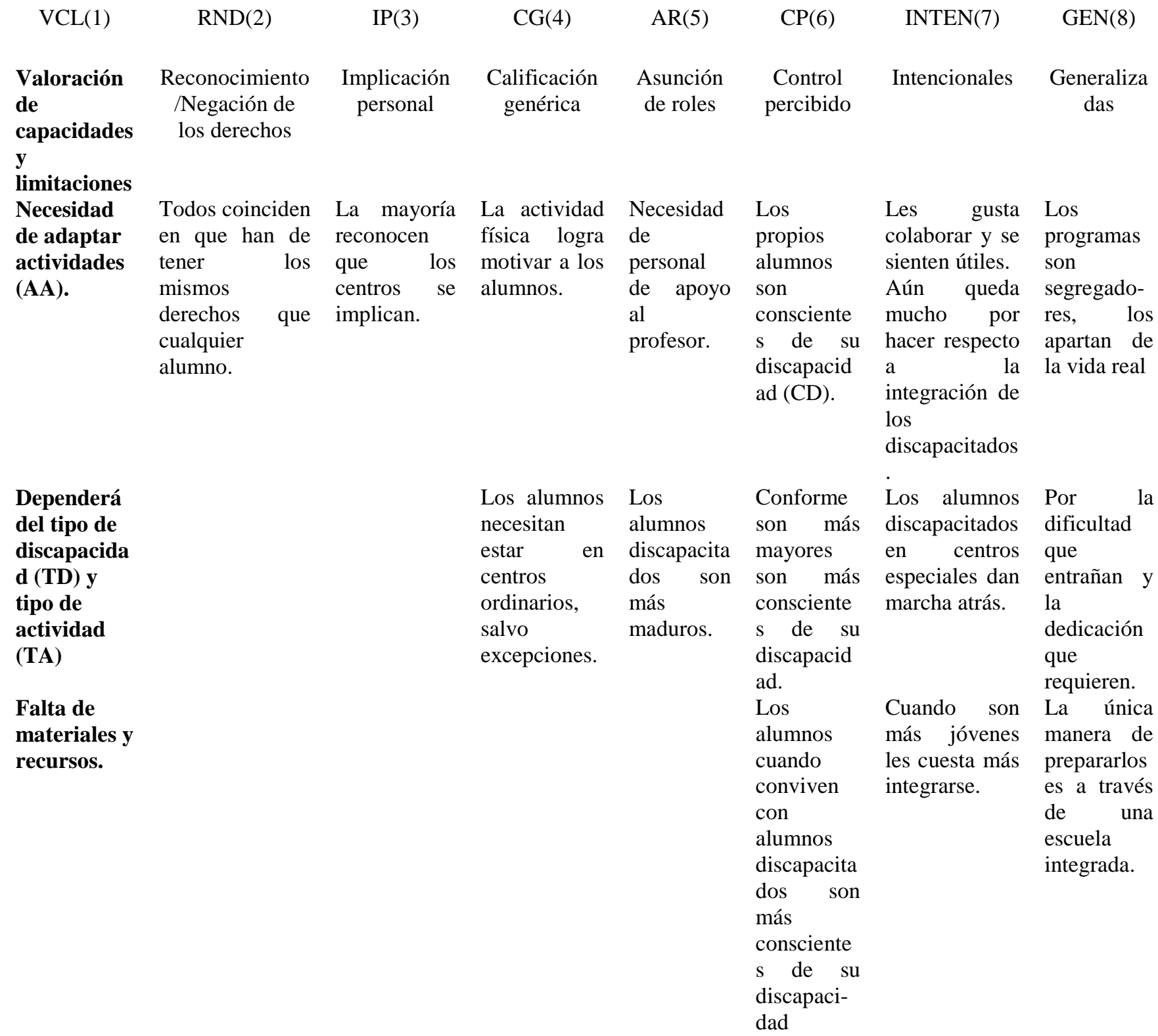

\section{Comunica-}


La variable INTEN (intencionalidad), pone de manifiesto que hay varias opiniones al respecto, ya que hay profesores que opinan que a los iguales les gusta colaborar y sentirse útiles ayudando y/o colaborando con sus compañeros, otros dicen que conforme son más pequeños les cuesta más integrarse en las clases. Hay diversidad de opiniones respecto a las actitudes mostradas en clase, pero en general determinan que en el $90 \%$ de los casos son actitudes positivas. Las últimas dos variables, GEN (generalizadas) hacen referencia al sistema educativo, y a los programas que se han llevado o se están realizando para favorecer la integración de los alumnos discapacitados en los centros docentes.

De todas las entrevistas (ver Tabla 5) se extraen principalmente las mismas conclusiones: a) se hacen necesarios mejoras en los planes educativos; b) que los alumnos deben estar en centros ordinarios para lograr una mejor integración; c) los profesores son conscientes de que es un trabajo duro, y que queda mucho por hacer; d) es necesario realizar reformas que enfocadas a los centros ordinarios. Se observa que es necesario mejorar en aspectos como la necesidad de llevar a cabo mejores programas educativos que ayuden a fomentar esa integración, tan importante para la mejoría y avance de los alumnos discapacitados con sus iguales. Por parte del personal docente, se evidencia también que se percibe una mayor nivel de integración por parte de los iguales, que son los propios alumnos, los iguales, los que contribuyen a favorecer y /o lograr dicha integración.

\section{Discusión}

Según los profesionales del entorno educativo encuestado (profesores de E.F y fisioterapeutas), no serían los iguales los causantes de esta falta de integración, si no el sistema educativo y las carencias que este acarrean para poder lograr la necesaria integración de los alumnos discapacitados en las aulas, y en concreto, de las clases de educación física.

En el presente estudio, se evidencia por parte de los responsables de la función docente (figura 5), la importancia que se considera que tiene la práctica de E.F en la integración de los alumnos discapacitados con sus iguales.

La realización de actividad física logra motivar a todos los alumnos (discapacitados y no discapacitados) en la mayoría de casos, logrado gracias a que dichos alumnos participan y/o colaboran en clase con los demás alumnos, por lo que se convierte de especial importancia que los discapacitados estén en centros ordinarios, (salvo casos graves), ya que les facilitará su integración y mejoría en las aulas, y así lograr un sentimiento de pertenencia dentro grupoclase.

Cuando se habla del proceso de inclusión-integración, todos los esfuerzos parecen centrarse en los primeros dos niveles, dejando de lado el tercer nivel del desarrollo prosocial y actitudinal ante la discapacidad.

Es importante resaltar, como conforme aumentan la edad y creencias de los compañeros, las actitudes hacia la discapacidad disminuyen. Coincidiendo con estudios anteriores (Gómez e Infante, 2004; Hammond et al, 2014), es muy importante reconocer que -entendiendo todas las dificultades existentes y posibles-, en los entornos educativos seleccionados, no se consigue ese desarrollo prosocial de los compañeros hacia la discapacidad del que hablan profesores y personal de apoyo. 


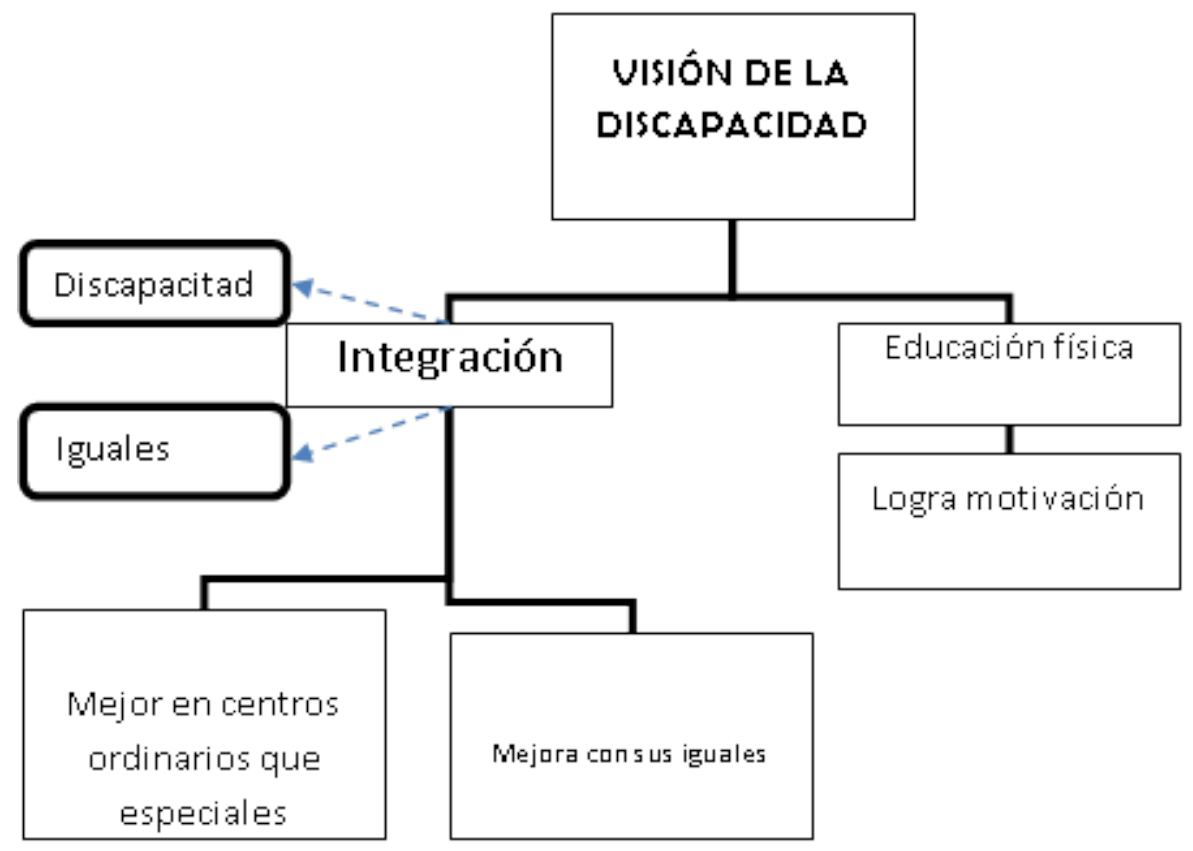

Figura 5. La importancia de la E.F en la integración de los alumnos discapacitados en las escuelas.

Este desarrollo actitudinal hacia el componente prosocial del individuo, se genera en cada una de las estructuras marcadas por estudios realizados con anterioridad (Novo-Corti et al., 2011), y atiende cognitiva, afectiva y comportamentalmente. Los datos del presente trabajo, no confirman que se generen habilidades a nivel actitudinal, aunque si cognitivo (creencias) entre compañeros, por lo que marca claramente la necesidad de un largo camino por hacer en este ámbito.

De forma más específica, los datos confirman diferencias significativas a favor de las chicas en cuanto a la capacidad de implicarse con los compañeros discapacitados, incluso a la hora de valorar sus capacidades y/o negación de derechos. Sin embargo, los chicos indican creencias a favor de actuar de una forma correcta e integradora hacia sus compañeros discapacitados, pero sin embargo, no lo manifiestan en las clases, en definitiva, lo piensan pero no hacen nada al respecto.

Es importante resaltar los datos que evidencian que los años de convivencia con alumnos discapacitados favorecen las actitudes y creencias de los compañero/as, llegando al máximo nivel en el tercer año de convivencia con los mismos. Por tanto, cuanto más tiempo pasan juntos discapacitados y compañeros, más aumentan sus actitudes, creencias, valoración personal, etc., hasta que llegado un momento, disminuye por completo todo el desarrollo actitudinal.

Estos mismos datos ponen de manifiesto, que a medida que los alumnos pasan más tiempo en las aulas con los alumnos discapacitados más aumentan sus creencias respecto a la integración de los mismos en las aulas, sin embargo, los datos muestran que a mayor nivel de creencias menores niveles de actuación y actitudes al respecto. 


\section{Referencias}

Aguado, A. L., Alcedo, M. A. y Arias, B.B. (2008). Cambio de actitudes hacia la discapacidad con escolares de primaria. Psicothema, 20(4), 697-704.

Alves, M. L. T., y Duarte, E. (2014). Students with disability? s perceptions about inclusion in Physical Education classes: a case study. Revista Brasileira de Educação Física e Esporte, 28(2), 329-338.

An, J., y Meaney, K. S. (2015). Inclusion Practices in Elementary Physical Education: A Social-cognitive Perspective. International Journal of Disability, Development and Education, 62(2), 143-157.

Araya-Cortés, A., González-Arias, M., y Cerpa-Reyes, C. (2014). Attitude of University Students Towards the Disabled. Educación y Educadores, 17(2), 289-305.

Bebetsos, E., Derri, V., Filippou, F., Zetou, E., y Vernadakis, N. (2014). Elementary School Children's Behavior towards the Inclusion of Peers with Disabilities, in Mainstream Physical Education Classes. Procedia-Social and Behavioral Sciences, 152, 819-823.

Bossaert, G., Colpin, H., Pijl, S. J., y Petry K. (2010). The attitudes of Belgian adolescents towards peers with disabilities. Research in Developmental Disabilities, 32, 504-509.

Bossaert, G., Colpin, H., Pijl, S. J., yPetry, K. (2013). Truly included? A literature study focusing on the social dimension of inclusion in education. International Journal of Inclusive Education, 17(1), 60-79.

Braga, L., Tracy, J. F., y Taliaferro, A. R. (2015). Physical Activity Programs in Higher Education: Modifying Net/Wall Games to Include Individuals with Disabilities. Journal of Physical Education, Recreation and Dance, 86(1), 16-22.

Calvo, C. O., Pérez-Tejero, J., y López, J. C. (2015). Propuesta de un programa de intervencióneducativa para facilitar la inclusión de alumnos con discapacidad en educación física. Retos, 27, 140-145.

Caus N., y Santos E. (2011).Análisis de la labor docente en el proceso de inclusión de alumnado con discapacidad en el área de educación física. Factors influencing the task of physical education teacher when disabledstudents are included in regular class. Educación y Diversidad, 5(1), 119-130.

Coates, J., y Vickerman, P. (2013). A review of methodological strategies for consulting children with special educational needs in physical education. European Journal of Special Needs Education, 28(3), 333-347.

Cologon, K. (2013). Inclusion in education. Towards equality for students with disability. Children and disability Australia. Australia

DePauw, K. P., y Doll-Tepper, G. (2000). Toward progressive inclusion and acceptance: Myth or reality? The inclusion debate and bandwagon discourse. Adapted Physical Activity Quarterly, 17, 135-143.

Echeita, G. y Ainscow, M. (2004). Escuelas inclusivas. Cuadernos de Pedagogía, 331, 49-80.

Florez García, M. A., Aguado Díaz, A. L., y Alcedo Rodríguez, M. A. (2009). Revisión y análisis de los programas de cambio de actitudes hacia personas con discapacidad. Anuario de Psicología Clínica y de la Salud. 5, 85-98. 
Futornyi, S. M. (2013). Ways to improve the organization of physical education students in higher education. Pedagogics, Psychology, Medical-biological Problems of Physical Training and Sports, 12, 94-100.

Gómez, V., e Infante, M. (2004). Actitudes de los estudiantes de educación hacia la integración de personas con discapacidad y hacia la educación multicultural. Cultura y Educación, 4, 371-383.

González, J., y Baños, L.M. (2012). Estudio sobre el cambio de actitudes hacia la discapacidad en clases de educación física. Cuadernos de Psicología del Deporte, 12, 101-108.

Goodwin, D. L., y Watkinson, E.J. (2000). Inclusive physical education from the perspective of students with physical disabilities. Adapted Physical Activity Quarterly, 17, 144-160.

Hammond, A. M., Young, J. A., y Konjarski, L. (2014). Attitudes of Australian Swimming Coaches towards Inclusion of Swimmers with an Intellectual Disability: An Exploratory Analysis. International Journal of Sports Science and Coaching, 9(6), 1425-1436.

Jeong, M., y Block, M. E. (2011). Physical education teachers' beliefs and intentions toward teaching students with disabilities. Research quarterly for exercise and sport, 82(2), 239-246.

Ko, B., y Boswell, B. (2013). Teachers' Perceptions, Teaching Practices, and Learning Opportunities for Inclusion. Physical Educator, 70(3), 223.

Madrid, D., Sánchez, P.,y García, D., (2011). De la exclusión a la inclusión: Una forma de entender y atender a la diversidad funcional en las instituciones escolares. Educación y Diversidad, 5(1), 23-31.

Molina, J., y Valenciano, J. (2010). Creencias y actitudes hacia un profesor de educación física en silla de ruedas: un estudio de caso. Revista de Psicología del Deporte,19(1), 137-149.

Muntaner, J. (2000). La igualdad de oportunidades en la escuela de la diversidad. Revista de Profesorado, 4(1), 27-45.

Muñoz-Cantero, J. M., Novo-Corti, I., y Rebollo-Quintela, N. (2013). Análisis de las actitudes, de los jóvenes trabajadores del sector textil hacia la discapacidad: diferencias por razón de género. Revista de Investigación Educativa, 31(1), 93-115.

Novo-Corti, I., Muñoz, J.,y Calvo, C. (2011). Análisis de las actitudes de los jóvenes universitarios hacia la discapacidad: un enfoque desde la teoría de la acción razonada. Relieve, 17(2),[Recuperado el 20 de marzo de 2015 http://www.uv.es/RELIEVE/v17n2/RELIEVEv17n2_5.htm].

Obrusnikova, I., y Dillon, S. R. (2012). Students' Beliefs and Intentions to Play with Peers With Disabilities in Physical Education: Relationships With Achievement and Social Goals. Journal of Teaching in Physical Education, 31(4), 311-328.

Özer, D., Nalbant, S., Ağlamıș, E., Baran, F., KayaSamut, P., Aktop, A., y Hutzler, Y. (2013). Physical education teachers' attitudes towards children with intellectual disability: the impact of time in service, gender, and previous acquaintance. Journal of Intellectual Disability Research, 57(11), 1001-1013.

Park, S. S., Koh, Y., y Block, M. (2014). Contributing Factors for Successful Inclusive Physical Education. Palaestra, 28(1).

Pierre Garel, J. (2007). Educación física y discapacidades motrices. Indepublicaciones. Barcelona. 
Place, K., y Hodge, S. R. (2001). Social inclusion of students with physical disabilities in general physical education: A behavior analysis. Adapted Physical Activity Quarterly, 18, 389-404.

Qi, J., y Ha, A. S. (2012). Inclusion in Physical Education: A review of literature. International Journal of Disability, Development and Education, 59(3), 257-281.

Sosa L. M. (2009). Educación física y discapacidad. Ágora para la EF y el Deporte, 9, 7-14.

Sullivan, E., y Masters, L. (2014). Changing Attitudes Toward Disabilities Through Unified Sports. Intellectual and Developmental Disabilities, 52(5), 367-378.

Tripp, A., Rizzo, T.L., and Webbert, L. (2007). Inclusion in Physical Education: Changing the Culture. Journal of Physical Education. Recreation and Dance, 78(2), 32-48. 